\title{
Differential context effects between sweet tastes and smells
}

\author{
Richard J. Stevenson and Mehmet Mahmut \\ Macquarie University, Sydney, New South Wales, Australia
}

\begin{abstract}
"Sweet" smells and tastes are perceptually similar, and physiological data indicate some commonality of central processing. However, sweet tastes and sweet smells do not provide interchangeable contexts in psychophysical experiments. The same sweet tastes are perceived as less intense when stronger sweet tastes are present, and they are perceived as more intense when weaker sweet tastes are present, as with sweet smells. However, complementary sets of sweet tastes and smells (e.g., weak sweet tastes, strong sweet smells) do not eliminate these differential context effects (DCEs). The present experiments examined, first, whether DCEs between sweet tastes and smells arise because of differences between odors and tastes in the way that sweetness scales with intensity as concentration rises, and, second, whether DCEs may be smaller for sweet tastes and smells, when contrasted with sweet tastes and nonsweet smells. The findings were clear: DCEs were consistently present, suggesting they are independent of perceptual similarity. These results imply that DCEs are probably not psychological in origin or centrally based; rather, they may have a subcortical locus.
\end{abstract}

Multisensory interaction that occurs between taste and smell during ingestion-flavor-seems to have consequences that are detectable at a later point in time, when the olfactory component of the flavor is smelled alone (Prescott, 1999; Small, 2008). For example, experiencing an odor mixed with a sweet taste later results in that odor's coming to be judged as more sweet smelling than a control odor experienced in water or another taste (e.g., Prescott \& Murphy, 2009; Stevenson, Prescott, \& Boakes, 1995; Yeomans \& Mobini, 2006). Such odorinduced tastes have now been studied in some depth, the main issue being the degree to which the taste component resembles, both perceptually and physiologically, taste sensations generated by physically present tastants. Perceptually, sweet smells may act to increase the judged intensity of a sweet taste to which they have been added (e.g., Frank \& Byram, 1988), and the magnitude of this "enhancement effect" is dependent on the degree to which the odor smells sweet (e.g., Stevenson, Prescott, \& Boakes, 1999; Valentin, Chrea, \& Nguyen, 2006). Moreover, sweet tastes may selectively facilitate the detection of sweet smells, further suggesting perceptual similarity (e.g., Dalton, Doolittle, Nagata, \& Breslin, 2000). Sweet tastes and sweet smells may also be confused in memory, such that later attempts to recall the frequency with which different taste and smell events occurred are conflated if the taste and odor are judged to smell alike (Stevenson \& Oaten, 2010). Finally, sweet smells can also speed the identification of sweet tastes and slow the identification of sour ones (White \& Prescott, 2007). Together, these findings have been taken to suggest that sweet tastes and the sweetness generated by certain odorants seem to be perceptually similar.

Further series of studies have begun to explore whether sweet tastes and smells rely on overlapping neural systems. Evidence is starting to emerge that suggests such an overlap in central processing. Stevenson, Miller, and Thayer (2008) found that participants with centrally impaired taste perception-insula lesions (primary taste cortex) - were also impaired in their ability to perceive the taste-like component of an odor. More recently, using neuroimaging, Veldhuizen, Nachtigal, and Small (2009) reported that the anterior insula is activated when smelling sweet odors, suggesting again neural overlap between brain areas involved in taste processing and brain areas involved in odor-induced taste perception. A further finding that also suggests neural commonality shows that sweet odors can produce a degree of pain tolerance comparable to that of sweet tastes, independent of any hedonic effect (Prescott \& Wilkie, 2007). So far, then, it would seem that odor-induced tastes share some central neural commonality with taste processing.

One finding that appears to yield results that are inconsistent with the data described above concerns differential context effects (DCEs). DCEs were first explored by Marks and colleagues (e.g., Marks, 1988, 1994; Marks \& Warner, 1991) in the auditory domain. The basic design of these experiments (see Figure 1 for an illustrative example relevant to the present article) involves exposing participants to two sets of stimuli. In early studies using the auditory modality, both sets of stimuli varied in sound pressure level (SPL) over the same range; however, in

R. J. Stevenson, dick.stevenson@mq.edu.au 
one condition, the low/moderate SPL stimuli were all of one frequency (e.g., $500 \mathrm{~Hz}$ ), and the moderate/high SPL stimuli were of another (e.g., $2500 \mathrm{~Hz}$ ). In the other condition, this relationship was reversed. Of interest here are participants' loudness judgments of the stimuli of similar (moderate) SPL. Namely, are they judged to be of similar loudness, or are they differentially affected by the context (i.e., only the sounds of corresponding frequency) in which they were presented? In these auditory studies, Marks and Warner found that where the two frequencies were similar, falling within an auditory band (e.g., fre- quencies of 1103 and $1134 \mathrm{~Hz}$ ), there was no DCE; that is, common SPL stimuli were judged to be of similar loudness. However, when the frequency difference was larger (e.g., 500 vs. $2500 \mathrm{~Hz}$ ), a DCE was evident. That is, the common SPL stimuli were judged as louder when quieter stimuli of the same frequency predominated and judged as quieter when louder stimuli of the same frequency predominated. On the basis of these and other findings, Rankin and Marks (1991) suggested that qualitative similarity between stimuli within a set might be important in moderating the DCE.

\section{Experimental Procedure}

All participants complete Conditions A and B

Condition A: Judge the intensity of...

Concentration Levels Administered

Odors (Low Context) Tastes (High Context)
Condition B: Judge the intensity of...

$$
\begin{aligned}
& \text { Concentration Levels Administered } \\
& \text { Odors (High Context) Tastes (Low Context) }
\end{aligned}
$$

\begin{tabular}{ll}
1 & \\
2 & 3 \\
3 & 4 \\
4 & 5 \\
5 & 6 \\
& 7 \\
\hline
\end{tabular}

\begin{tabular}{ll} 
& 1 \\
& 2 \\
3 & 3 \\
4 & 4 \\
5 & 5 \\
6 & \\
7 & \\
\hline
\end{tabular}

\section{Possible Outcomes}

Intensity ratings for the common (gray

highlighted) stimuli show whether...

A differential context effect is observed.

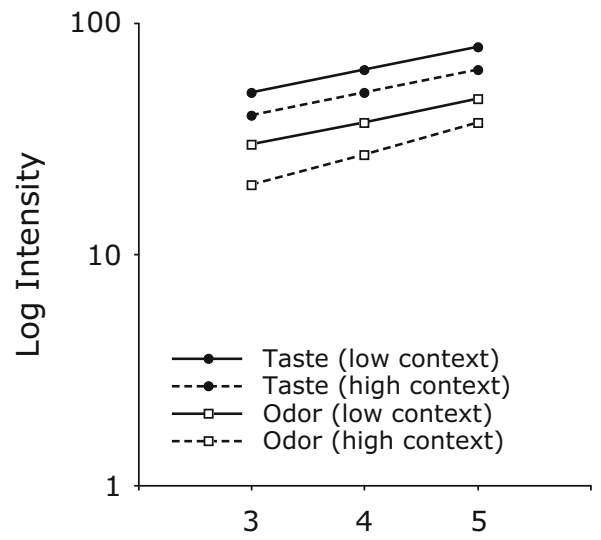

Concentration Level
A differential context effect is not observed.

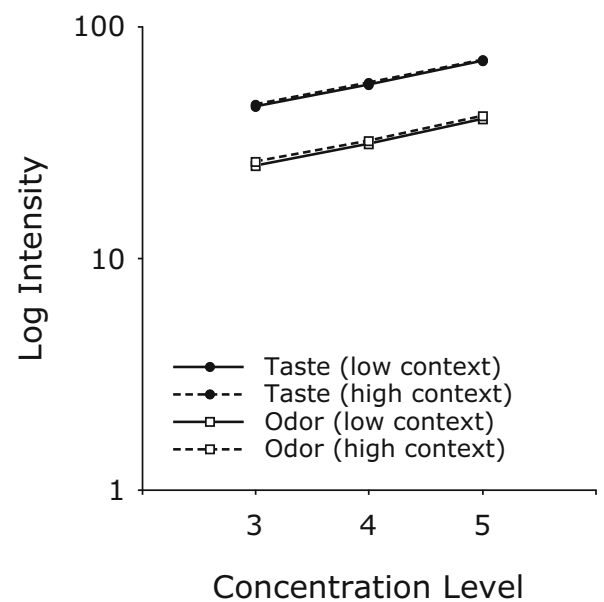

Figure 1. Diagrammatic illustration of the basic experimental procedure and the possible outcomes for a differential context experiment (in this example, using tastes and smells). 
DCEs have also been explored in the chemical senses (Rankin \& Marks, 1991, 2000). With tastes, sucrose and sodium chloride are dissimilar, and DCEs are readily evident (Rankin \& Marks, 1991). However, when mixtures of sucrose and sodium chloride are used (i.e., increasing perceptual similarity), the DCE is eliminated. In a subsequent study, Rankin and Marks (2000) examined whether DCEs occurred when perceptually similar taste (sucrose) and olfactory (sweet-smelling) stimuli were used. Whether the sweet-smelling odors were sniffed or sampled orally (retronasal olfactory perception) had little bearing on the outcome. DCEs were readily evident, even though participants reported a high degree of perceptual similarity between these stimuli.

If DCEs are typically eliminated when stimuli are perceptually similar, but they still occur for sweet tastes and odor-induced sweet tastes, this would suggest one of two mutually exclusive conclusions. The first is that tastes and odor-induced tastes are not, in fact, perceptually similar; rather, their apparent similarity may instead reflect semantic knowledge. This would suggest a very different interpretive framework (i.e., one based on knowledge of odortaste relationships) than the view currently predominant in the literature (i.e., one based on automatic activation of the taste system by a smell) (Stevenson \& Tomiczek, 2007; Verhagen \& Engelen, 2006). The ramifications of such a conclusion would extend beyond perception into associative learning. It has been claimed that odor-taste conditioning, which seems to underpin odor-induced tastes, may be one of the few instances of learning without awareness. This is a disputed claim (see, e.g., Lovibond \& Shanks, 2002), and findings that suggest a semantic basis for odorinduced tastes would lend support to the view that participants do, in fact, learn odor-taste associations explicitly.

The second and alternative conclusion would be that DCEs are not automatically eliminated when the stimulus sets are perceptually similar. Instead, a different factor, of which perceptual similarity is sometimes a correlate, would be responsible. This different factor could relate to the neural level (on a dimension of peripheral to central) at which DCEs occur, favoring, in this alternative conclusion, a more peripheral locus (Arieh \& Marks, 2002). A more peripheral locus for these effects could then, in some instances, coincide with perceptual similarity (as with critical bands in audition or taste types), but, in other cases, not (perhaps as with tastes and odorinduced tastes).

It is important that which of these mutually exclusive conclusions proves to be correct depends on whether Rankin and Marks's (2000) study provided an adequately robust test of the effects of perceptual similarity. If there were effects of perceptual similarity and these had been missed in their studies, this would eliminate any conflict between their findings and current thinking about the perceptual nature of odor-induced tastes. Relatedly, it would also imply a more central locus for DCEs. This is because perceptual similarity, at least for odors and tastes, probably reflects more central (cortical) processing, as recent data would suggest (Prescott \& Wilkie,
2007; Stevenson et al., 2008; Veldhuizen et al., 2009). In contrast, were a more detailed study to find that perceptual similarity exerts no effect on DCEs, this would imply that Rankin and Marks's (2000) findings do not bear on the question of perceptual similarity between tastes and odor-induced tastes. Furthermore, it would suggest that the mechanism responsible for this type of context effect is moderated by a process in a more peripheral location.

For these reasons, the observation of DCEs between sweet tastes and sweet smells (stimuli that appear to be perceptually similar) would seem to warrant more detailed scrutiny. In Experiments 1A and 1B, we examined whether an underlying assumption in the Rankin and Marks (2000) study might be incorrect. For tastants such as sucrose, concentration changes are likely to result in parallel alterations in intensity and quality (sweetness). Crudely, more sugar in a drink makes it both stronger tasting and sweeter. This intensity-quality coupling is almost certainly different for odors. Whereas the range of potential odor intensities might be similar to the range of taste intensities, perceived sweetness for a particular odorant may not demonstrate a similar range. This could occur for many reasons, from receptor-level binding effects to the multitude of qualities that are perceived when most odors are smelled. At its extreme, this might lead to little change in sweetness, since odor intensity increases with concentration, resulting in different levels of similarity between the taste and smell across concentration. This, then, might account for why Rankin and Marks (2000) still observed a DCE for stimuli that are apparently perceptually similar. Experiment $1 \mathrm{~A}$ investigated whether odor sweetness ratings are less tightly coupled to changes in intensity as concentration increases, relative to sweet tastes. Experiment 1B tested whether artificially coupling odor intensity and sweetness, by selecting different odorants that varied on these parameters, would reduce or eliminate a DCE with sucrose. Experiment 2 set out to test a further possibility. Since both our Experiment 1B and Rankin and Marks's (2000) series of studies used just sweet smells and tastes, perhaps the magnitude of the DCE would be even more substantial if one compared nonsweet smells and sweet tastes. That is, these earlier studies might be hiding evidence of a similarity effect by failing to compare these results with a more dissimilar control condition.

\section{EXPERIMENT 1A}

Experiment 1A had two aims. First, we wished to test two related hypotheses concerning intensity-quality coupling in sweet tastes and odors. We suspected that, for odors, the correlation between intensity and sweetness across concentration would be significantly weaker than the correlation between intensity and sweetness for sucrose. We also suspected that (1) changes in sweetness between the highest and lowest odor concentrations and (2) changes in intensity between the same would have a ratio (i.e., Change Score 1 divided by Change Score 2) 
lower than unity and lower than the comparable ratio for sucrose. If supported, these findings would suggest that changes in odor concentration for sweet-smelling odors do not lead to equivalent changes in intensity and sweetness - an underlying assumption for Rankin and Marks's (2000) original series of studies.

The second aim of Experiment 1A was to select a set of odors and tastes varying in sweetness and intensity that could then be used in Experiment 1B. In particular, we wanted to obtain a set of stimuli where the intensity of the odor increased in an approximately linear manner with its sweetness and where this range of sweetness and intensity was similar to the set of sweet tastes also judged in this experiment. This would create a set of odor stimuli where the coupling of quality (sweetness) and intensity is artificially equivalent to the sweet taste used here, sucrose.

\section{Method}

Participants. Twelve healthy adult participants ( 8 female, 4 male; mean age $=26.0$ years) took part for course credit. All were naive about psychophysical experiments and had never participated in an olfactory-related study.

Stimuli. Ten sweet solutions were prepared by diluting sucrose in tap water to the following concentrations: $34.2 \%(1.0 \mathrm{M}), 25.0 \%$ (0.73 M), 18.5\% (0.54 M), 13.4\% (0.39 M), 9.9\% (0.30 M), $7.2 \%$ $(0.21 \mathrm{M}), 5.1 \%(0.15 \mathrm{M}), 3.8 \%(0.11 \mathrm{M}), 2.7 \%(0.08 \mathrm{M})$, and $2.1 \%$ $(0.06 \mathrm{M})$. Sucrose was presented in aliquots of $10 \mathrm{ml}$ in disposable plastic cups. Forty-two odorant samples were prepared by initially diluting the odorant in propylene glycol, and then with tap water, to the requisite concentration (see Table 1). Odorants were presented as 20 -ml solutions in transparent screw-top jars.

Procedure. The study comprised four components split over 2 consecutive days - taste intensity judgments, odor intensity judgments, taste sweetness judgments, and odor sweetness judgmentswith each stimulus being rated once within each component. Presentation order of these four components was randomized for each participant. Participants were instructed on the use of the labeled magnitude scale (LMS; Green et al., 1996) — notably, that they could respond anywhere along its length and that they were to judge the samples relative to that of the top anchor ("strongest sensation of any kind" [see Bartoshuk et al., 2004]). For taste intensity judgments, participants poured the solution into their mouths and then expectorated. They then rated the intensity of the stimulus on the LMS (question: "How strong?"). This was followed by a water rinse and a 20 -sec interstimulus interval (ISI). Stimulus presentation order was randomized for each participant. On completing these judgments, there was a 5-min break before the next component began. For odor intensity judgments, participants sniffed three times over the jar containing the odorant and then rated its intensity on the LMS scale (question: "How strong?"). A 20 -sec ISI separated each trial. Odor stimuli were presented in a different quasi-random order for each participant ("quasi-random" because an odor was never allowed to follow itself). The procedures for the taste sweetness and odor sweetness judgments were identical in all but two respects. First, different randomized presentation orders were employed. Second, sweetness ratings were obtained for each stimulus, again using the LMS (question: "How sweet?").

\section{Results and Discussion}

Two hypotheses were tested using these data. First, we calculated the mean Pearson correlation coefficient between intensity and sweetness ratings for the odors (calculated by participant for each odor and then averaged across odors to generate one correlation for each participant) and for sucrose. The mean intensity-sweetness correlation for sucrose was .85 (this value was .93 if only the weakest, middle, and strongest concentrations were used), and for the odors, it was .63. After using Fisher's transformation, the two sets of correlations were compared using a paired samples $t$ test. This revealed that the relationship between intensity and sweetness was stronger for sucrose $\left(r^{\prime}=1.48, S D=0.65\right)$ than for the sweet-smelling odors $\left(r^{\prime}=0.81, S D=0.29\right)$ $[t(11)=3.04, p<.01]$. Second, we tested whether the ratio of (1) mean change in sweetness between the highest and lowest odor concentration and (2) mean change in intensity between the same was lower than unity for odors and lower than the same ratio for sucrose. Odors had a mean ratio of $0.84(S D=0.19)$, which was significantly lower than unity $[t(11)=2.91, p<.02]$ and significantly lower than that for sucrose $(M=1.32, S D=0.47)[t(11)=4.36, p<$ $.001]$. These findings suggest that, on average, sweetness ratings for odors are less tightly coupled to ratings of intensity, as concentration changes, than are sweetness ratings for sucrose (see Figure 2). Although these findings do support our initial concern, they also rule out its more extreme forms (i.e., no or very weak coupling), because sweetness judgments here were clearly correlated with intensity as concentration increased.

Table 1

Odorants, Sources, and Concentrations

for Stimuli Used in Experiment $1 \mathrm{~A}$

\begin{tabular}{llll}
\hline \multicolumn{1}{c}{ Odorant (Source) } & \multicolumn{3}{c}{ Concentration Steps (\%) } \\
\hline Kiwi (Dragoco) & $2.5 \times 10^{-2}$ & $2.5 \times 10^{-3}$ & $2.5 \times 10^{-5}$ \\
Aniseed (Quest) & $2.0 \times 10^{-3}$ & $2.0 \times 10^{-5}$ & $1.0 \times 10^{-6}$ \\
Citral (Dragoco) & $7.5 \times 10^{-4}$ & $7.5 \times 10^{-6}$ & $7.5 \times 10^{-8}$ \\
Eugenol (Sigma) & $1.0 \times 10^{-3}$ & $1.0 \times 10^{-5}$ & $5.0 \times 10^{-7}$ \\
Limonene (Sigma) & $2.0 \times 10^{-3}$ & $2.0 \times 10^{-5}$ & $1.0 \times 10^{-6}$ \\
Carvone (Dragoco) & $1.0 \times 10^{-3}$ & $1.0 \times 10^{-5}$ & $5.0 \times 10^{-7}$ \\
Plum (Quest) & $2.0 \times 10^{-3}$ & $2.0 \times 10^{-5}$ & $2.0 \times 10^{-7}$ \\
Amyl acetate (Sigma) & $1.0 \times 10^{-3}$ & $1.0 \times 10^{-5}$ & $2.5 \times 10^{-7}$ \\
Sweetness enhancer (Dragoco) & $1.5 \times 10^{-2}$ & $1.5 \times 10^{-3}$ & $1.5 \times 10^{-5}$ \\
Cherry (Quest) & $2.0 \times 10^{-3}$ & $2.0 \times 10^{-5}$ & $2.0 \times 10^{-7}$ \\
Strawberry (Quest) & $2.0 \times 10^{-3}$ & $2.0 \times 10^{-5}$ & $2.0 \times 10^{-6}$ \\
Vanilla (Dragoco) & $2.0 \times 10^{-2}$ & $2.0 \times 10^{-3}$ & $2.0 \times 10^{-5}$ \\
Ethyl butyrate (Sigma) & $1.8 \times 10^{-3}$ & $1.8 \times 10^{-5}$ & $4.4 \times 10^{-7}$ \\
Caramel (Dragoco) & $2.5 \times 10^{-3}$ & $2.5 \times 10^{-5}$ & $2.5 \times 10^{-7}$ \\
\hline
\end{tabular}




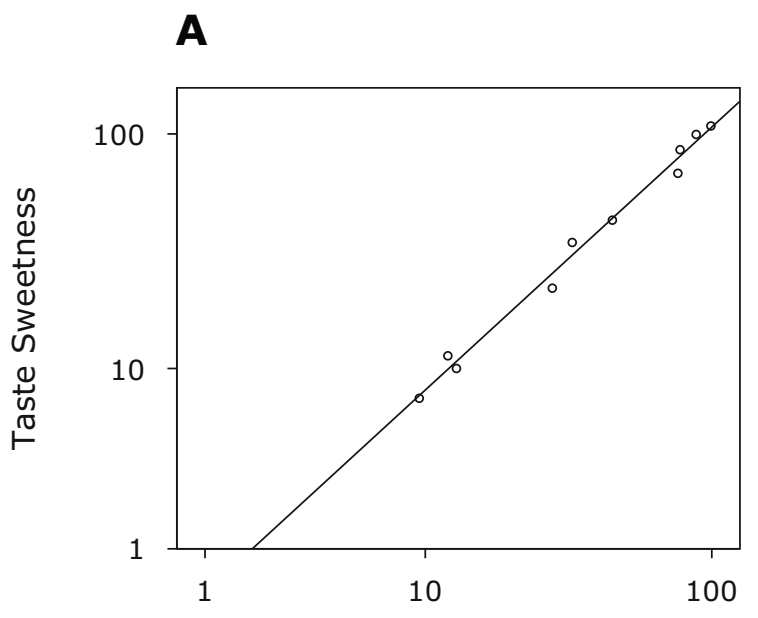

Taste Intensity

B

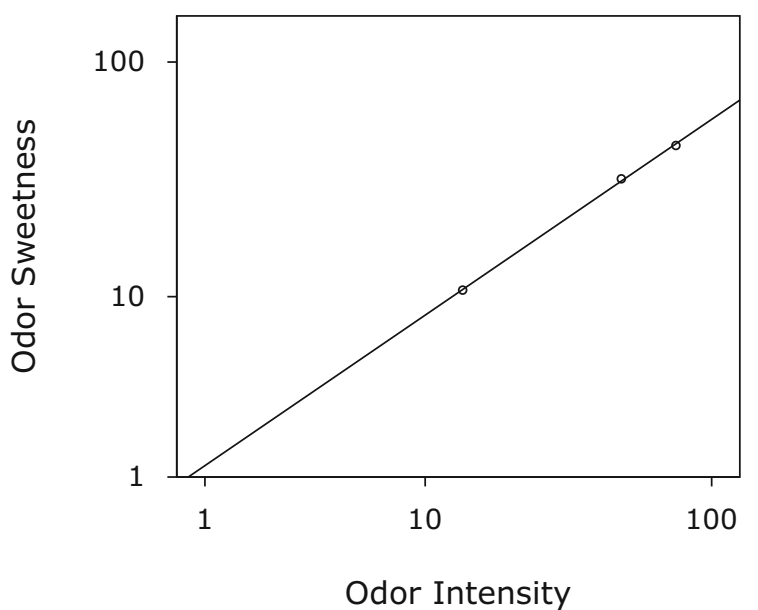

Figure 2. (A) Log scatterplot of geometric mean intensity ratings against geometric mean sweetness ratings for the sucrose concentration series. (B) Log scatterplot of geometric mean intensity ratings (collapsed across odor type) against mean sweetness ratings (collapsed across odor type) for the three concentration levels employed.

Stimuli were initially selected for Experiments $1 \mathrm{~A}$ and $1 \mathrm{~B}$ on the basis of an examination of means. These selections were then tested using ANOVA to ensure that intensity and sweetness ratings for the tastes and odors were parallel across concentration levels of sucrose. The selected stimuli, along with their intensity and sweetness ratings, are presented in Table 2. A three-way repeated measures ANOVA on the intensity and sweetness ratings (log transformed) of the final selection was conducted with rating type (sweetness vs. intensity), modality (taste vs. smell), and concentration (seven levels) as the within-participants factors. This revealed only one significant effect [concentration: $\left.F(6,66)=56.70, M S_{\mathrm{e}}=0.11, p<.001\right]$, with intensity and sweetness ratings increasing linearly with concentration [linear trend: $F(1,11)=159.1]$ to the same extent for both odors and tastes. This analysis confirms that the odors and tastes to be used in Experiment 1B reflect a similar degree of coupling between sweetness and intensity, thereby minimizing any difference in qualitative similarity (at least for sweetness) across concentration.

\section{EXPERIMENT 1B}

The aim of this experiment was to see whether the DCE would still be present when the stimulus set developed in Experiment 1A was employed - that is, one that minimizes any difference in qualitative similarity between odor and taste across concentration (see Figure 1 for an explanatory diagram of the basic procedure used here and its possible outcomes). Ratings were obtained here for both intensity and sweetness to test whether any observed DCE would be smaller when sweetness judgments were made, on the basis that this rating would likely act to maximize the chance of participants' judging the odors and tastes within the same context.

\section{Method}

Participants. Thirty-six healthy adult participants (27 female, 9 male; mean age $=20.1$ years) took part for course credit. All were naive about psychophysical experiments and had never participated in an olfactory-related study.

Stimuli. Odor and taste stimuli for this experiment are detailed in Table 2.

Procedure. Participants attended two experimental sessions 1 week apart. Half of the participants received the 5 strongest sweet tastes and the 5 weakest odor stimuli in Session 1 and the 5 weakest sweet tastes and the 5 strongest odor stimuli in Session 2 and vice versa for the other half of the participants. In each session, the order of stimulus presentation was randomized. There were two replicates of the stimulus set tested in each session. In one replicate, participants rated intensity using the LMS scale for all 10 stimuli, and, in the other replicate, they rated sweetness for all 10 stimuli using the LMS scale (as described in Experiment 1A). The rating that was used first was counterbalanced across participants. The procedure in each trial was as follows: (1) For tastes, participants were asked to pour the solution into their mouths, expectorate, rate the stimulus, rinse, and then wait $30 \mathrm{sec}$ before commencing the next trial; (2) for odors, participants took three sniffs of the stimulus, made their rating, and waited for $30 \mathrm{sec}$ before commencing the next trial.

\section{Results}

The log transformed intensity ratings were analyzed using a three-way ANOVA with stimulus (odor vs. sucrose), condition (high vs. low), and concentration (three levels) as within-participants factors. It is both important and consistent with Rankin and Marks (2000) that we observed a DCE (see Figure 3A), with a significant interaction between stimulus and condition $\left[F(1,35)=15.29, M S_{\mathrm{e}}=\right.$ $0.14, p<.001]$. The common sucrose solutions were judged to be around $9 \%$ more intense when weaker sucrose solutions were present (see Figure 3A) than when more concentrated sucrose solutions were present. Similarly for the odors, the common odor solutions were judged to be $8 \%$ more intense when weaker odors were present (see Figure $3 \mathrm{~A}$ ) than when stronger odors were present. There was also a main effect of stimulus $\left[F(1,35)=26.95, M S_{\mathrm{e}}=\right.$ $0.38, p<.001]$, with odors judged as less intense than tastes; a main effect of concentration $[F(2,70)=82.75$, 
Table 2

\begin{tabular}{|c|c|c|c|c|c|c|c|c|c|}
\hline \multicolumn{10}{|c|}{$\begin{array}{l}\text { Geometric Means and Standard Deviations of Intensity } \\
\text { and Sweetness Ratings for the Selected Set of Tastants } \\
\text { and Odorants From Experiment } 1 \mathrm{~A} \text { and Used in Experiment 1B }\end{array}$} \\
\hline \multicolumn{5}{|c|}{ Sucrose } & \multicolumn{5}{|c|}{ Odorant } \\
\hline \multirow{2}{*}{$\begin{array}{c}\text { Concentration } \\
\text { Level }(\%)\end{array}$} & \multicolumn{2}{|c|}{ Intensity } & \multicolumn{2}{|c|}{ Sweetness } & \multirow{2}{*}{$\begin{array}{c}\text { Concentration } \\
\text { Level }(\%)\end{array}$} & \multicolumn{2}{|c|}{ Intensity } & \multicolumn{2}{|c|}{ Sweetness } \\
\hline & $M$ & $S D$ & $M$ & $\overline{S D}$ & & $M$ & $S D$ & $M$ & $S D$ \\
\hline 2.7 & 13.0 & 4.4 & 10.0 & 4.0 & ET $4.4 \times 10^{-7}$ & 11.1 & 4.2 & 9.0 & 4.1 \\
\hline 3.8 & 12.1 & 4.8 & 11.4 & 3.9 & KW $2.5 \times 10^{-5}$ & 13.2 & 4.0 & 10.1 & 3.7 \\
\hline 5.1 & 28.4 & 4.0 & 22.5 & 3.9 & $\mathrm{AN} 1.0 \times 10^{-6}$ & 33.5 & 4.1 & 23.4 & 4.2 \\
\hline 7.2 & 33.3 & 3.8 & 35.2 & 3.8 & $\mathrm{CM} 2.5 \times 10^{-5}$ & 36.7 & 4.1 & 38.5 & 4.3 \\
\hline 9.9 & 45.8 & 4.0 & 43.8 & 3.9 & PL $2.0 \times 10^{-5}$ & 39.8 & 3.7 & 45.2 & 3.8 \\
\hline 13.4 & 76.7 & 3.7 & 68.5 & 3.8 & $\mathrm{CH} 2.0 \times 10^{-3}$ & 77.1 & 3.6 & 72.9 & 4.0 \\
\hline 18.5 & 78.0 & 3.6 & 85.9 & 3.7 & $\mathrm{SE} 1.5 \times 10^{-2}$ & 91.4 & 3.6 & 83.8 & 3.9 \\
\hline
\end{tabular}

Note-ET, ethyl butyrate; KW, kiwi; AN, aniseed; CM, caramel; PL, plum; CH, cherry; SE, sweetness enhancer.

$\left.M S_{\mathrm{e}}=0.14, p<.001\right]$; and a stimulus $\times$ concentration interaction $\left[F(2,70)=9.77, M S_{\mathrm{e}}=0.15, p<.001\right]$, with a greater disparity between odor and taste intensity ratings for the weakest stimuli.

The log transformed sweetness ratings were analyzed in the same manner, and the results were broadly the same. There was evidence of a DCE (see Figure 3B), with a significant interaction between stimulus and condition $\left[F(1,35)=6.31, M S_{\mathrm{e}}=0.13, p<.02\right]$, the common sucrose solutions being judged as $6 \%$ less sweet when more concentrated solutions were present (see Figure 3B) and odors being judged as $6 \%$ less sweet when more concentrated odorants were present (see Figure 3B). Main effects of stimulus $\left[F(1,35)=36.04, M S_{\mathrm{e}}=0.24, p<.001\right]$ and concentration $\left[F(2,70)=93.32, M S_{\mathrm{e}}=0.15, p<.001\right]$ and a stimulus $\times$ concentration interaction $[F(2,70)=$ $\left.16.11, M S_{\mathrm{e}}=0.13, p<.001\right]$ were also observed, with similar meaning as above.

Finally, we examined whether the stimulus $\times$ condition interaction differed by type of rating scale. To test this, we collapsed across concentration and calculated a mean DCE for taste and for smell when sweetness and intensity ratings were used. These data were then analyzed using a two-way repeated measures ANOVA with stimulus (taste vs. odor) and rating (sweetness vs. intensity) as withinparticipants factors. The ANOVA revealed no main effect or interaction involving rating $\left(F_{\mathrm{S}}<1\right)$, and, as might be expected, a main effect of stimulus $[F(1,35)=15.79$, $\left.M S_{\mathrm{e}}=1.04, p<.001\right]$, indicating that the magnitude of the DCE was unaffected by the type of rating employed.

\section{Discussion}

The DCE was still present even when we used a stimulus set that was designed to match sweetness and intensity across different odor and taste concentrations. This procedure closely matched those characteristics, but it is important to note that these odors had other qualities, as well as probable trigeminal impact, that may have detracted from the qualitative similarity between sucrose and the odors used here. However, the finding that the type of rating made (intensity or sweetness) had no significant effect on the outcome suggests that, even when attention is explicitly drawn to the commonality between the differ- ent stimuli (with the sweetness rating), there was still no reduction in the size of the DCE.

It is interesting to note that this DCE, although highly reliable, was smaller than that observed by Rankin and Marks (2000). Intensity changes for sucrose were around $9 \%$, whereas, for the two most comparable experiments in Rankin and Marks (2000, Experiments 3 and 4), the values were $30 \%$ and $37 \%$, respectively. Obviously, there were procedural differences in our design, and these could account for some of the observed discrepancy, but it is noteworthy that perhaps the most obvious difference-the range of sucrose concentrations employed - was, in fact, much greater in one of their experiments (relative to ours) and much weaker in another. However, both yielded similarly sized shifts in intensity ratings. Perhaps, then, our experiment here does indicate a reduction in the size of the DCE under conditions where there was arguably greater perceptual similarity between the tastes and smells than in the Rankin and Marks (2000) study. One way to test this assertion more directly is to contrast DCEs for sweet and nonsweet odors. If better matching of the range of sweetness intensities between odors and tastes did, in fact, act to reduce the magnitude of the DCE, then this effect should be most evident when two sets of odors are used: one well matched qualitatively to sucrose and the other not. In Experiment 2, we set out to test this hypothesis.

\section{EXPERIMENT 2}

In Experiment 2, we tested whether a DCE would be smaller when a sweet-smelling odor was used than when a nonsweet-smelling odor was used. The design was similar to that in Experiment 1A in all but four ways. First, the same sweet-smelling odorant and the same nonsweetsmelling odorant served at all concentration levels because we expected the difference in similarity to be sufficiently large between odors to overwhelm any effect of the weaker quality-intensity coupling in this modality. Second, we chose to use just intensity ratings in this experiment because there was no difference by rating type in Experiment $1 \mathrm{~B}$. In addition, sweetness ratings would have had little applicability to a nonsweet-smelling odor. Third, we used a between-participants design with odor type (sweet 


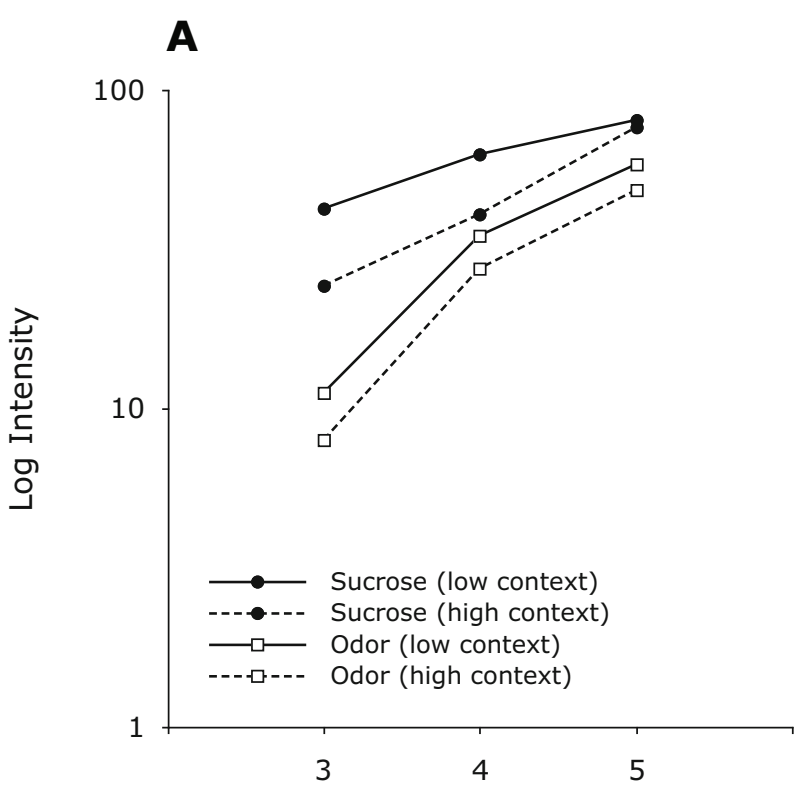

Concentration Level

B

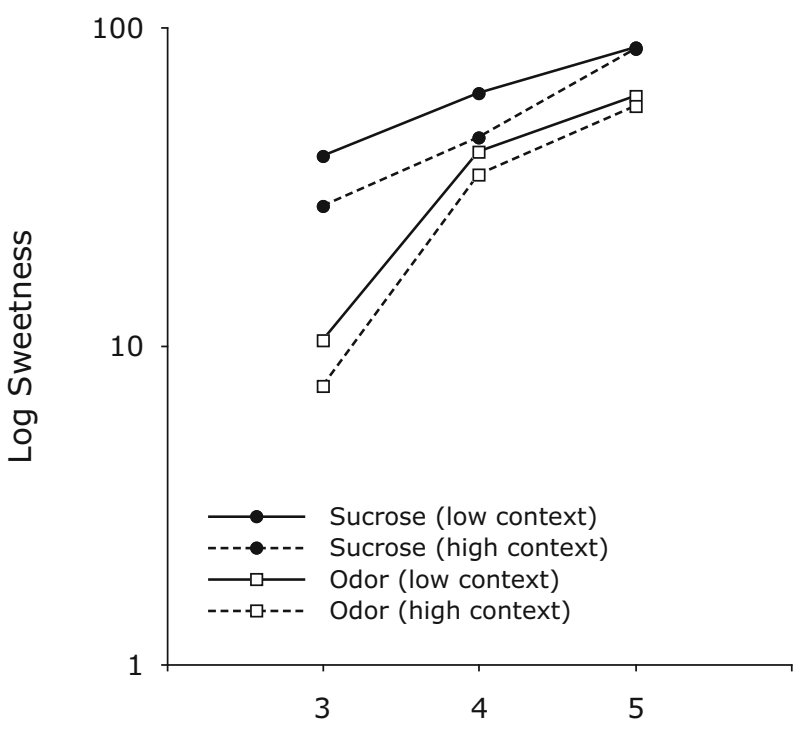

Concentration Level

Figure 3. (A) Log plot of geometric mean intensity ratings for the same three concentrations of sucrose and odor, under conditions in which the sucrose series included only additional weaker sucrose stimuli (sucrose low context), only additional stronger sucrose stimuli (sucrose high context), only additional weaker odor stimuli (odor low context), or only additional stronger odor stimuli (odor high context). (B) As above, except here, the geometric mean sweetness ratings are presented.

vs. nonsweet) as the between-participants factor and with the contextual manipulation as a within-participants factor, so as to limit the experiment to two sessions. Finally, after participants completed the study, we had them make similarity judgments between (1) the lowest, middle, and highest concentrations of each odorant and (2) the middle concentration of sucrose, to check that the expected similarity difference was apparent.

\section{Method}

Participants. Twenty-eight healthy adult participants ( 23 female, 5 male; mean age $=21.8$ years) took part for course credit. These participants were randomly allocated to group by order of arrival to the study. All were naive about psychophysical experiments and had never participated in an olfactory-related study.

Stimuli. A pilot experiment $(n=14)$ was used to generate odor (sweet, nonsweet) and taste stimuli that were matched for intensity (using the same ANOVA approach to test this as reported in Experiment $1 \mathrm{~A}$ ) and to confirm that the sweet-smelling odor was judged suitably similar to sucrose and the nonsweet-smelling odor was judged suitably dissimilar. Taste stimuli were the same as those described in Experiment 1B (see Table 2). The sweet-smelling odor was vanilla (Dragoco), which was initially dissolved in propylene glycol and then serially diluted with tap water to the following concentrations: $5.0 \times 10^{-2 \%}, 1.7 \times 10^{-2 \%}, 5.6 \times 10^{-3 \%}, 1.9 \times 10^{-3 \%}$, $6.2 \times 10^{-4 \%}, 2.1 \times 10^{-4 \%}$, and $6.8 \times 10^{-5 \%}$. The nonsweetsmelling odor was terpineol (Sigma, smelling of disinfectant), which was prepared in the same manner as the vanilla, at the following concentrations: $2.0 \times 10^{-2 \%}, 6.7 \times 10^{-3 \%}, 2.2 \times 10^{-3} \%, 7.5 \times$ $10^{-4 \%}, 2.5 \times 10^{-4 \%}, 8.2 \times 10^{-5 \%}$, and $2.7 \times 10^{-5 \%}$. It should be noted that terpineol also has greater irritant properties than does vanilla, adding to the qualitative difference between these two odorants. Stimuli were presented as in Experiment 1B.

Procedure. Participants attended two experimental sessions that were 1 week apart. Half of the participants in the sweet-odor group received the five strongest sweet-taste and the five weakest sweetodor stimuli in Session 1 and the five weakest sweet-taste and the five strongest sweet-odor stimuli in Session 2, and vice versa for the other half of the participants in the sweet-odor group. The same procedure was adopted for the other group but, this time, using the nonsweet odor. In each session, the order of stimulus presentation was randomized. There were two replicates tested in each session, but the same rating (intensity) was made on the LMS scale (as for Experiment 1A) each time, and the two ratings averaged for the analysis. The procedure in each trial was as described in Experiment 1B, again using a 30 -sec ISI. Finally, participants were asked to sip and spit the central concentration of sucrose, and then, in randomized order, to sniff both the sweet and nonsweet odor at the weakest, the middle, and the strongest concentration of the series. In each case, they were asked to judge the similarity of the odor to the sweet taste they had sampled at the start of these trials on a seven-point category scale that ranged from $1=$ not at all to $7=$ very.

\section{Results}

The log transformed intensity ratings were analyzed using a four-way mixed design ANOVA with stimulus (odor vs. sucrose), condition (high vs. low), and concentration (three levels) as within-participants factors and group (sweet vs. nonsweet odors) as the betweenparticipants factor. We observed a DCE with a significant interaction between stimulus and condition $[F(1,26)=$ $\left.17.53, M S_{\mathrm{e}}=0.05, p<.001\right]$. The common sucrose solutions were judged to be $10 \%$ more intense when weaker sucrose solutions were present (see Figures 4A and 4B) than when more concentrated sucrose solutions were present. Similarly for the odors, the common odor solutions were judged to be around $10 \%$ more intense when weaker 

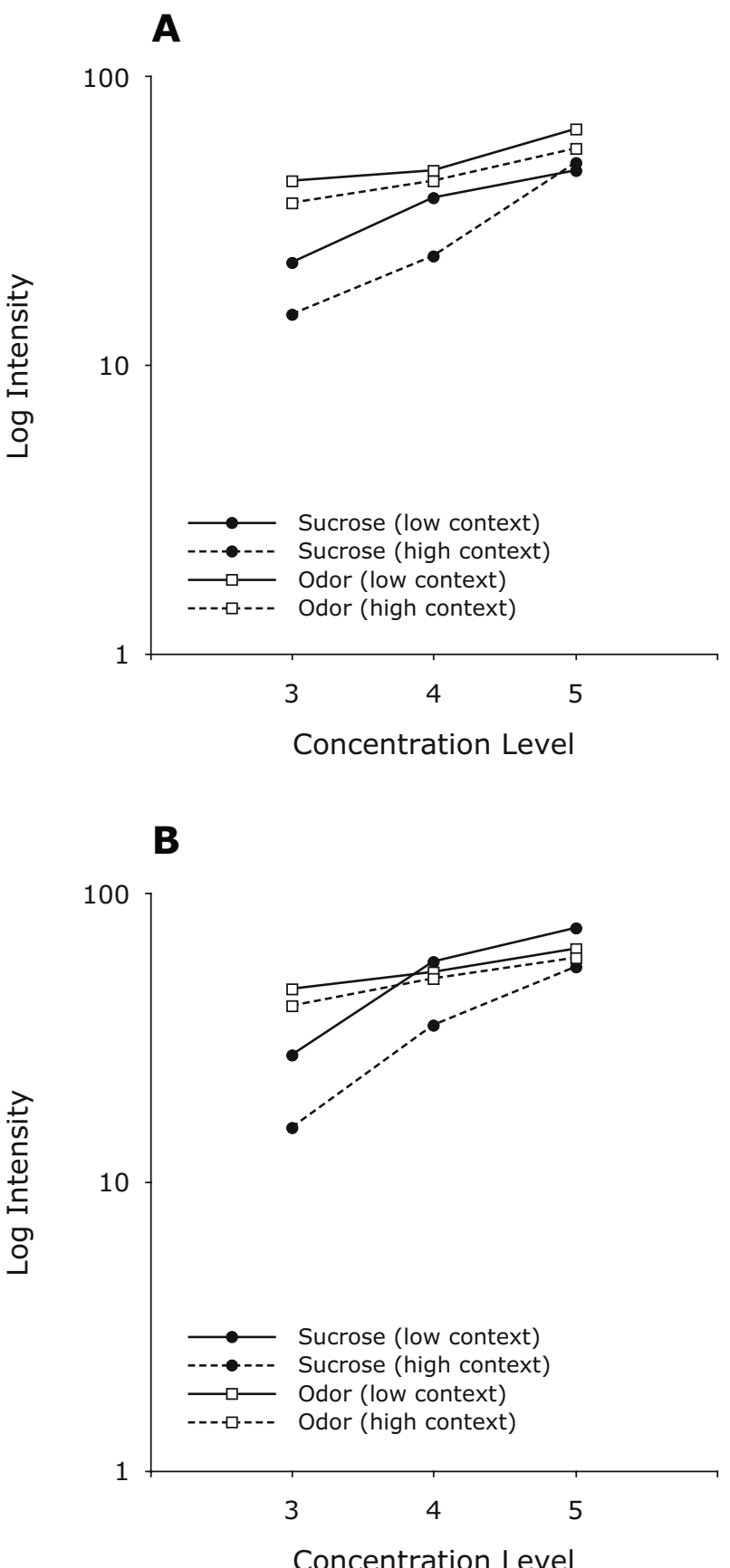

Figure 4. (A) Log plot of geometric mean intensity ratings for the same three concentrations of sucrose and odor, under conditions in which the sucrose series included only additional weaker sucrose stimuli (sucrose low context), only additional stronger sucrose stimuli (sucrose high context), only additional weaker sweet odor stimuli (odor low context), or only additional stronger sweet odor stimuli (odor high context). (B) As above, except here, a nonsweet odor was used.

odors were present than when stronger odors were present. Because these effects are comparable in magnitude to those observed in Experiment 1B, this suggests that procedural differences between Rankin and Marks's (2000) study and our own probably account for the smaller effect size here-especially since our DCE was completely unaffected by the between-groups manipulation of odor/ taste similarity $(F<1$ for the stimulus $\times$ condition $\times$ group interaction). Also, there were not any other effects involving group in the analysis (see Figures 4A and 4B). There was also a small stimulus $\times$ condition $\times$ concentration interaction $\left[F(2,52)=4.89, M S_{\mathrm{e}}=0.02, p<.02\right]$, with the DCE varying slightly by concentration: larger for sucrose with the middle concentration and smaller for the odors on the comparable concentration. Finally, there were main effects of stimulus $\left[F(1,26)=10.63, M S_{\mathrm{e}}=\right.$ $0.21, p<.005]$, with odors judged as more intense than tastes, and of concentration $\left[F(2,52)=69.21, M S_{\mathrm{e}}=\right.$ $0.05, p<.001]$, and a stimulus $\times$ concentration interaction $\left[F(2,52)=18.93, M S_{\mathrm{e}}=0.03, p<.001\right]$, with a greater disparity between odor and taste intensity ratings for the weakest stimuli.

Similarity ratings (to sucrose) for the sweet and nonsweet odors were analyzed in a three-way mixed design ANOVA with odor (sweet vs. nonsweet) and concentration (weakest vs. central vs. strongest) as within-participants factors and group (used sweet or nonsweet odors in the earlier part of the experiment) as a between-participants factor. The ANOVA revealed a significant effect of odor $\left[F(1,26)=92.44, M S_{\mathrm{e}}=2.56, p<.001\right]$, with vanilla being judged as substantially more similar to sucrose $(M=$ $3.9 / 7)$ than was the nonsweet odor terpineol $(M=1.5 / 7)$. There was also a main effect of concentration $[F(2,52)=$ $\left.10.59, M S_{\mathrm{e}}=1.31, p<.001\right]$ and an interaction between concentration and odor $\left[F(2,52)=13.01, M S_{\mathrm{e}}=1.24\right.$, $p<.001]$. For the latter, whereas similarity ratings remained largely the same for terpineol $(M \mathrm{~s}=1.4,1.5$, 1.6), the central concentration of vanilla was judged more similar to sucrose $(M=5 ; p s<.05$; Bonferroni-adjusted post hoc contrasts) than the weakest $(M=3.8)$ and strongest $(M=2.9)$ concentrations. Nonetheless, vanilla was still judged as more similar to sucrose than was terpineol at each concentration step (all $t \mathrm{~s}>4.1$ ). Finally, there was one effect involving group and an interaction with concentration $\left[F(2,52)=3.98, M S_{\mathrm{e}}=1.31, p<.05\right]$, but since this effect did not involve odor and was unexpected, we suggest that this effect is of little import.

\section{GENERAL DISCUSSION}

The studies reported here suggest that the DCE for qualitatively similar (and dissimilar) tastes and smells is a highly robust effect. Using a carefully matched set of sweet odors and tastes in Experiment 1B and directly manipulating the similarity of the taste and smells in Experiment 2 had no discernible effect on the DCE. In our introduction, we suggest that Rankin and Marks's (2000) findings might be problematic for the current view that tastes are perceptually similar to odor-induced tastes. The new data reported here suggest that DCEs are not related to perceptual similarity and therefore have no bearing on this argument. Instead, as we discuss below, our findings suggest a subcortical locus for these context effects. 
The data from the two experiments reported here provide two reasons for thinking that perceptual similarity is unrelated to the presence or absence of DCEs for tastes and odor-induced tastes. The first and arguably most compelling reason is that when we used odor-taste combinations that differed significantly in perceived similarity (i.e., vanilla [sweet-smelling]-sucrose vs. terpineol [disinfectant-smelling]-sucrose), the DCE was present to the same degree for both sets of stimuli. This would suggest that similarity between tastes and smells is not related to the magnitude of the DCE and, thus, that DCEs have no bearing on whether tastes and odor-induced tastes are perceptually similar. The second reason is that when odor and taste stimuli were closely matched - arguably providing more ideal circumstances to generate a common judgmental context - and even when the commonality between the taste and the smells was drawn to participants' attention by the sweetness rating, this also had no effect on the DCE. Together, these results and those from other studies of DCEs in the chemical senses (i.e., Rankin \& Marks, 1991) suggest that perceptual similarity is a sometime correlate of another process that actually dictates whether stimulus magnitude of one set of stimuli is affected by another. For reasons described below, we suggest that this process may be located above the level of the receptors but below that of central cortical-level processing.

Determining the locus of the context effects observed here is complicated by the fact that the neural architecture of olfaction is significantly different from that of the other senses. Olfaction has no obligatory thalamic relay (it makes direct projections to the limbic system), and primary processing occurs in paleocortical tissue (e.g., Cahill \& McGaugh, 1998; Shepherd, 1974; Tham, Stevenson, \& Miller, 2009). Taste and olfactory information only start to converge at the cortical level, so processing is largely independent up to this point. Cortical coprocessing seems to involve the insular (Stevenson et al., 2008; Veldhuizen et al., 2009) and orbitofrontal cortices, although the data supporting the latter site (Rolls \& Baylis, 1994) are drawn from animals rather than from people. These structures represent primary and secondary taste cortex (and, in the case of the orbitofrontal cortex, secondary olfactory cortex). Since these structures seem to be the neural correlates of taste and certain olfactory percepts, thus forming the basis for any perceptual commonalities, it would seem unlikely that intensitive context effects are based at this level in the brain. If they were, perceptual similarity would likely eliminate or reduce such effects; but, as the data here indicate, it does not.

If a cortical locus is unlikely, then one alternative is a receptor-based locus. However, this seems unlikely too, since the context effects observed here - and by Rankin and Marks (2000) - are successive rather than simultaneous and therefore suggest a processing capacity that stores and averages information and then increases the gain for low-magnitude stimuli and decreases the gain for high-magnitude stimuli, an adaptation-like process (see Schneider \& Parker, 1990). This type of processing is probably beyond that of the taste and olfactory recep- tors alone. If this alternative then appears unlikely, as with a central cortical locus, this would imply a subcortical locus. Olfaction has at least two plausible locations that could accommodate the type of adaptation-like process that Schneider and Parker envisaged. The first is the olfactory bulb, and the second is the piriform paleocortex. Both structures are known to support adaptation in animals, and both are sufficiently complex as to be able to store and average information (Best \& Wilson, 2004; Scott, 1977). With regard to taste, the nucleus of the solitary tract (NST) seems to be a likely candidate structure, since it too may support adaptation, and some work, again in animals, suggests that it may be sensitive to the context in which a stimulus is presented (Di Lorenzo \& Lemon, 2000; Smith, Liu, \& Vogt, 1996). Moreover, input to the NST has both receptor-specific channels (i.e., fibers preferentially carrying information from one receptor type) and multireceptor channels (i.e., fibers carrying information from multiple receptor types), which might account for DCEs with pure tastants and their elimination with taste mixtures (see Rankin \& Marks, 1991). In the latter case, perceptual similarity between taste mixtures is simply a correlate of other processes that, in fact, govern whether or not a DCE occurs.

An additional issue that the data here touch upon is the linkage between intensity and quality. For tastes, such as sucrose, sweetness and intensity seem to be closely coupled. For odors, the intensitive and qualitative dimensions are more loosely coupled, with the magnitude of the quality changing less with intensity than for taste. Part of this difference may be accounted for by the fact that, as odorant concentration at the receptor layer increases, the range of receptors that are activated will also increase (Malnic, Hirono, Sato, \& Buck, 1999). This, then, may produce alterations in the perceptual quality of the stimulus (e.g., Gross-Isseroff \& Lancet, 1988; Laing, Legha, Jinks, \& Hutchinson, 2003). Such changes in odor quality as odor concentration changes may well account for some of the weaker coupling we observed in Experiment 1A. A further possibility concerns the nature of odor quality versus the nature of taste quality. Odor qualities are numerous and seem to rely, at least to some extent, on prior experience (e.g., Li, Luxenberg, Parrish, \& Gottfried, 2006; Stevenson, 2001). In the case of odor-induced tastes, this reliance on past experience is particularly well established (e.g., Stevenson, Boakes, \& Prescott, 1998). If an odor is smelled, and this reactivates in some way a prior taste experience, then how is that taste quality scaled with the magnitude of the odor concentration? Unfortunately, questions like this have not been well addressed in the literature, but they are surely important, since odor quality is far more diverse and experience dependent than taste quality.

In conclusion, the present experiments demonstrate that similarity between a taste and a smell is independent of the process that underpins the context effect evident in our experiments. This independence from perceptual similarity suggests that these context effects are not cortical phenomena, and we suggest that a subcortical locus is most likely. 


\section{AUTHOR NOTE}

The authors thank the Australian Research Council for their continuing support. Correspondence concerning this article should be addressed to R. J. Stevenson, Department of Psychology, Macquarie University, Sydney, NSW 2109, Australia (e-mail: dick.stevenson@mq.edu.au).

\section{REFERENCES}

Arieh, Y., \& Marks, L. E. (2002). Context effects in visual length perception: Role of ocular, retinal, and spatial location. Perception \& Psychophysics, 64, 478-492.

Bartoshuk, L. M., Duffy, V. B., Greene, B. G., Hoffman, H. J., Ko, C.-W., Lucchina, L. A., ET AL. (2004). Valid across-group comparisons with labeled scales: The gLMS versus magnitude matching. Physiology \& Behavior, 82, 109-114.

Best, A. R., \& WiLson, D. A. (2004). Coordinate synaptic mechanisms contributing to olfactory cortical adaptation. Journal of Neuroscience, 24, 652-660.

Cahill, L., \& McGaugh, J. L. (1998). Mechanisms of emotional arousal and lasting declarative memory. Trends in Neurosciences, 21, 294-299.

Dalton, P., Doolittle, N., Nagata, H., \& Breslin, P. A. S. (2000). The merging of the senses: Integration of subthreshold taste and smell. Nature Neuroscience, 3, 431-432.

Di Lorenzo, P. M., \& Lemon, C. H. (2000). The neural code for taste in the nucleus of the solitary tract of the rat: Effects of adaptation. Brain Research, 852, 383-397.

Frank, R. A., \& Byram, J. (1988). Taste-smell interactions are tastant and odorant dependent. Chemical Senses, 13, 445-455.

Green, B. G., Dalton, P., Cowart, B., Shaffer, G., Rankin, K., \& HigGins, J. (1996). Evaluating the "labeled magnitude scale" for measuring sensations of taste and smell. Chemical Senses, 21, 323-334.

Gross-IsserofF, R., \& LANCET, D. (1988). Concentration-dependent changes of perceived odor quality. Chemical Senses, 13, 191-204.

Laing, D. G., Legha, P. K., Jinks, A. L., \& Hutchinson, I. (2003). Relationship between molecular structure, concentration and odor qualities of oxygenated aliphatic molecules. Chemical Senses, 28, 57-69.

Li, W., Luxenberg, E., Parrish, T., \& Gottfried, J. A. (2006). Learning to smell the roses: Experience-dependent neural plasticity in human piriform and orbitofrontal cortices. Neuron, 52, 1097-1108.

Lovibond, P. F., \& SHAnks, D. R. (2002). The role of awareness in Pavlovian conditioning: Empirical evidence and theoretical implications. Journal of Experimental Psychology: Animal Behavior Processes, 28, 3-26.

Malnic, B., Hirono, J., Sato, T., \& Buck, L. B. (1999). Combinatorial receptor codes for odors. Cell, 96, 713-723.

MARKS, L. E. (1988). Magnitude estimation and sensory matching. Perception \& Psychophysics, 43, 511-525.

MarKs, L. E. (1994). "Recalibrating" the auditory system: The perception of loudness. Journal of Experimental Psychology: Human Perception \& Performance, 20, 382-396.

Marks, L. E., \& WARnER, E. (1991). Slippery context effect and critical bands. Journal of Experimental Psychology: Human Perception \& Performance, 17, 986-996.

PrescotT, J. (1999). Flavour as a psychological construct: Implications for perceiving and measuring the sensory qualities of foods. Food Quality \& Preference, 10, 349-356.

Prescott, J., \& Murphy, S. (2009). Inhibition of evaluative and perceptual odour-taste learning by attention to the stimulus elements. Quarterly Journal of Experimental Psychology, 62, 2133-2140.

Prescott, J., \& Wilkie, J. (2007). Pain tolerance selectively increased by a sweet smelling odor. Psychological Science, 18, 308-311.
RANKIN, K. M., \& MARKS, L. E. (1991). Differential context effects in taste perception. Chemical Senses, 16, 617-629.

RANKIN, K. M., \& MARKs, L. E. (2000). Chemosensory context effects: Role of perceived similarity and neural commonality. Chemical Senses, 25, 747-759

Rolls, E. T., \& BAYLIS, L. L. (1994). Gustatory, olfactory, and visual convergence within the primate orbitofrontal cortex. Journal of Neuroscience, 14, 5437-5452.

Schneider, B., \& PArker, S. (1990). Does stimulus context affect loudness or only loudness judgments? Perception \& Psychophysics, 48, 409-418.

ScotT, J. W. (1977). A measure of extracellular unit responses to repeated stimulation applied to observations of the time course of olfactory responses. Brain Research, 132, 247-258.

Shepherd, G. M. (1974). The synaptic organization of the brain. Oxford: Oxford University Press.

Small, D. M. (2008). Flavor and the formation of category-specific processing in olfaction. Chemosensory Perception, 1, 136-146.

Smith, D. V., LiU, H., \& Vogt, M. B. (1996). Responses of gustatory cells in the nucleus of the solitary tract of the hamster after $\mathrm{NaCl}$ or amiloride adaptation. Journal of Neurophysiology, 76, 47-58.

Stevenson, R. J. (2001). The acquisition of odour qualities. Quarterly Journal of Experimental Psychology, 54A, 561-577.

Stevenson, R. J., Boakes, R. A., \& Prescott, J. (1998). Changes in odor sweetness resulting from implicit learning of a simultaneous odor-sweetness association: An example of learned synesthesia. Learning \& Motivation, 29, 113-132.

Stevenson, R. J., Miller, L. A., \& Thayer, Z. C. (2008). Impairments in the perception of odor-induced tastes and their relationship to impairments in taste perception. Journal of Experimental Psychology: Human Perception \& Performance, 34, 1183-1197.

Stevenson, R. J., \& OAten, M. J. (2010). Sweet odours and sweet tastes are conflated in memory. Acta Psychologica, 134, 105-109.

Stevenson, R. J., Prescott, J., \& Boakes, R. A. (1995). The acquisition of taste properties by odors. Learning \& Motivation, 26, 433455

Stevenson, R. J., Prescott, J., \& Boakes, R. A. (1999). Confusing tastes and smells: How odours can influence the perception of sweet and sour tastes. Chemical Senses, 24, 627-635.

Stevenson, R. J., \& TomiczeK, C. (2007). Olfactory induced synesthesias: A review and model. Psychological Bulletin, 133, 294-309.

Tham, W. W. P., Stevenson, R. J., \& Miller, L. A. (2009). The functional role of the mediodorsal thalamic nucleus in olfaction. Brain Research Reviews, 62, 109-126.

Valentin, D., Chrea, C., \& Nguyen, D. H. (2006). Taste-odour interactions in sweet taste perception. In W. J. Spillane (Ed.), Optimising sweet taste in foods (pp. 66-84). Cambridge: Woodhead.

Veldhuizen, M. G., Nachtigal, D. J., \& Small, D. M. (2009). Taste cortex contributes to odor quality coding [Abstract]. Chemical Senses, 34, A8.

Verhagen, J. V., \& Engelen, L. (2006). The neurocognitive bases of human multimodal food perception: Sensory integration. Neuroscience \& Biobehavioral Reviews, 30, 613-650.

White, T. L., \& PrescotT, J. (2007). Chemosensory cross-modal Stroop effects: Congruent odors facilitate taste identification. Chemical Senses, 32, 337-341.

Yeomans, M. R., \& Mobini, S. (2006). Hunger alters the expression of acquired hedonic but not sensory qualities of food-paired odors in humans. Journal of Experimental Psychology: Animal Behavior Processes, 32, 460-466.

(Manuscript received April 4, 2010; revision accepted for publication June 17, 2010.) 